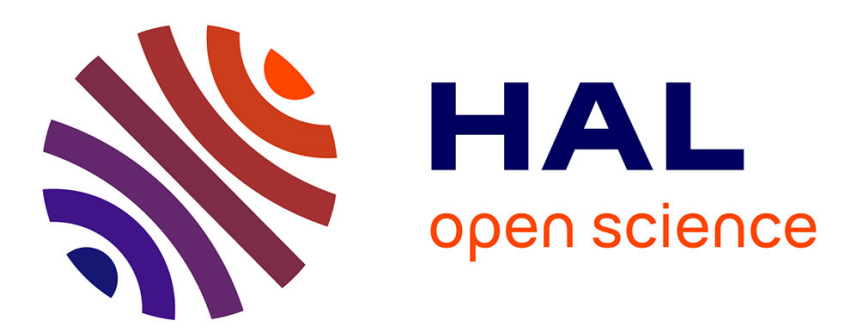

\title{
Oculomotor Impairments in Developmental Dyspraxia
}

B. Gaymard, M. Giannitelli, G. Challes, S. Rivaud-Péchoux, O. Bonnot, D. Cohen, J. Xavier

\section{To cite this version:}

B. Gaymard, M. Giannitelli, G. Challes, S. Rivaud-Péchoux, O. Bonnot, et al.. Oculomotor Impairments in Developmental Dyspraxia. The Cerebellum, 2017, 16 (2), pp.411 - 420. 10.1007/s12311016-0817-6 . hal-01496103

\section{HAL Id: hal-01496103 https://hal.sorbonne-universite.fr/hal-01496103}

Submitted on 27 Mar 2017

HAL is a multi-disciplinary open access archive for the deposit and dissemination of scientific research documents, whether they are published or not. The documents may come from teaching and research institutions in France or abroad, or from public or private research centers.
L'archive ouverte pluridisciplinaire HAL, est destinée au dépôt et à la diffusion de documents scientifiques de niveau recherche, publiés ou non, émanant des établissements d'enseignement et de recherche français ou étrangers, des laboratoires publics ou privés. 


\section{Oculomotor impairments in developmental dyspraxia}

Gaymard $B^{1}$, Giannitelli $\mathrm{M}^{2,5}$, Challes $\mathrm{G}^{3}$, Rivaud-Péchoux $\mathrm{S}^{4}$, Bonnot $\mathrm{O}^{2}$, Cohen $\mathrm{D}^{2,5}$, Xavier $\mathrm{J}^{2}$

${ }^{1}$ Service de Neurophysiologie Clinique

${ }^{2}$ Service de Psychiatrie de l'Enfant et de l'Adolescent

${ }^{3}$ Service d'Ophtalmologie

${ }^{4}$ Sorbonne Universités, UPMC Univ Paris 06, Inserm, CNRS, UM 75, U 1127,UMR 7225, ICM, F-75013

AP-HP, Hôpital de la Salpêtrière, 75651 Paris

FRANCE

${ }^{5}$ Institut des Systèmes Intelligents et Robotiques

Université Pierre et Marie Curie, 1 place Jussieu, 75005, Paris

FRANCE

Corresponding author:

Bertrand Gaymard. Hôpital de la Salpêtrière, Service de Neurophysiologie Clinique, AP-HP, Paris, France

Phone : +(33) 142163614

Email: bertrand.gaymard@gmail.com

The authors disclose any financial or commercial conflict of interest with this article. 


\begin{abstract}
Children with developmental dyspraxia (DD) express impairments in the acquisition of various motor skills and in the development of their social cognition abilities. Although the neural bases of this condition are not fully understood, they are thought to involve frontal cortical areas, subcortical structures and the cerebellum. Although cerebellar dysfunction is typically difficult to assess and quantify using traditional neurophysiological methods, oculomotor analysis may provide insight into specific cerebellar patterns. The aim of the present study was to investigate, in dyspraxic and typically developing subjects, various oculomotor saccade tasks specifically designed to reveal frontal and cerebellar dysfunction. In addition to evidence supporting prefrontal dysfunction, our results revealed increased variability of saccade accuracy consistent with cerebellar impairments. Furthermore, we found that dyspraxic patients showed decreased velocities of non-visually guided saccades. A closer analysis revealed significant differences in saccade velocity profiles with slightly decreased maximum saccade velocities, but markedly prolonged deceleration phases. We show that this pattern was not related to a decreased state of alertness, but was suggestive of cerebellar dysfunction. However, the clear predominance of this pattern in non-visuallyguided saccades warrants alternative hypotheses. In light of previous experimental and anatomical studies, we propose that this unusual pattern may be a consequence of impaired connections between frontal areas and cerebellar oculomotor structures.
\end{abstract}

Key Words: Saccades, skewness, developmental dyspraxia, cerebellum 


\section{Introduction}

Developmental dyspraxia (DD) is a heterogeneous disorder marked by several disabilities in motor and non-motor domains in the absence of clear neurological deficits [1]. Affected children display delayed acquisition of motor skills, particularly affecting body movement coordination (e.g., impaired balance, walking and running) and fine motor skills (e.g., impaired writing, catching balls). Cluster analyses of large samples of children with DD have distinguished two pure subtypes, ideomotor and visual-spatial/visual-constructional dyspraxia, and a mixed subtype, the latter being the most common [2]. ]. Children with DD also frequently exhibit non-motor difficulties such as in planning, organization and time management skills, and in language and social cognition abilities [3, 4].

Many studies have examined the affected processes and corresponding neural correlates of DD, the most frequently cited brain structures being the frontal lobes, basal ganglia, and cerebellum [5]. Because DD is associated with impaired motor adaptation, coordination and balance, the theory that the disorder is cerebellar in nature has been well supported. However, cerebellar dysfunction may be difficult to assess in individuals with subtle symptoms, especially in the absence of quantitative and specific measures.

In contrast to the somatomotor system, the oculomotor system provides an accurate and valid tool for the assessment of cerebellar function. All categories of eye movements involve cerebellar structures [6]. Decreased gain with phase lag of horizontal smooth pursuit $[7,8]$ and selective impairment of vertical smooth pursuit [9] have been reported. However, no pathological pattern of smooth pursuit impairment may be specifically ascribed to cerebellar dysfunction. Furthermore, smooth pursuit requires cooperation and attentional resources that may be dubious in young patients.

Conversely, our understanding of the cerebellar contribution to saccades is much more established, with precise saccade impairments specifically linked to cerebellar dysfunction [6]. Furthermore, saccades enable the targeting of higher cortical functions, especially those involving the frontal lobes [10]. Therefore, it is surprising that saccadic eye movements have not been investigated in dyspraxic children. A major role of the oculomotor cerebellum is the control of saccade accuracy and the maintenance of reduced variability of saccade amplitude on a trial-to-trial basis $[6,11]$. The aim of the present study was to analyze saccadic eye movements in a group of patients with DD in comparison to agedmatched typically developing (TD). In order to examine potential cerebellar disorders, we 
focused on means and variability of saccade amplitude. In order to probe frontal lobe function, we tested several saccade paradigms, with reflexive and volitional saccades, including an antisaccade task especially designed to evaluate the frontal lobe's ability to inhibit reflexive saccades [10]. Our study revealed disorders compatible with both frontal and cerebellar impairments and an unexpected and non-previously reported impairment of saccade velocities, mostly observed on non-visually guided saccades, on which the present paper is focused.

\section{Subjects and Methods}

\section{Participants}

Eleven adolescents with DD (6 boys, 5 girls; mean age: 17, SD: 2.8) were recruited in the Department of Child and Adolescent Psychiatry of Pitié-Salpêtrière Hospital, Paris. Patients were defined as having DD if they met the following DSM-5 (American Psychiatric Association, 2013) [12] criteria for Developmental Coordination Disorder (DCD - because developmental dyspraxia is included in DCD).

A psychomotor assessment, including the M-ABC [13], Bender [14], Frostig [15] and BHK [16] evaluations, was performed by an occupational therapist for all subjects with DD. We excluded patients with a known neurological condition or with verbal IQ $<70$. Given the high rate of comorbidity in subjects with dyspraxia, all patients were assessed using the Wechsler Intelligence Scale for Children or Adults according to age $[17,18]$.

Subjects with suspicion of autism spectrum disorder or other chronic psychiatric condition (e.g., schizophrenia) were assessed with the Autism Diagnostic Interview-Revised or the Diagnostic Interview for Genetic Studies 2, respectively. Table 1 provides the clinical characteristics of our dyspraxic sample. Each adolescent was individually matched according to age with a typically developing adolescent of the control group.

\section{Control group}

Eleven TD adolescents (4 boys, 7 girls; mean age: 17, SD: 2.9) were recruited via local high schools for participation in the TD group. Control subjects had no history of learning disabilities, behavior problems, visual perception difficulties, and/or other neuropsychiatric conditions. Cognitive assessments were not performed on the TD group, for which chronological age was used. In addition, chronological age corresponded to expected levels of scholastic achievement for the TD group. 
Written consent was received from all participants and/or their parents where appropriate. This study was approved by a local ethics committee and was in accordance with the Declaration of Helsinki.

\section{Oculomotor study}

Our study was initially designed to analyze visually guided saccades and antisaccades in children with DD. The observation of a particular velocity profile in antisaccades prompted us to add two paradigms: the delayed saccade task, i.e. a volitional visually guided saccade task, and the memory-guided saccade task, i.e. a volitional non-visually guided saccade task. Therefore, these two paradigms were performed only in 7 out of our 11 patients.

\section{- Setup}

Eye movements were recorded in a dark and quiet room. Subjects were seated in an armchair with the head stabilized by a chinrest and a forehead contact. Visual stimulations were displayed on an liyama monitor located $57 \mathrm{~cm}$ in front of them (visual angle: $56^{\circ} \times 35^{\circ}$ ). Horizontal and vertical eye movements were recorded with a video-based Eyetracker (Mobile EBTC, eyeBRAIN, www.eye-brain.com, France), at a sampling rate of $300 \mathrm{~Hz}$. Each session started with a calibration procedure in which subjects were asked to look at a target presented at 13 successive locations. The oculomotor tasks were controlled by the MeyeParadigm software. Data were stored and analyzed subsequently with the MeyeAnalysis software (both softwares: www.eye-brain.com, France). Saccade onset and offset were automatically detected by a velocity threshold criterion and systematically controlled by the experimenter. Saccades with a latency below $90 \mathrm{~ms}$ or altered by blinks were discarded.

A recording session consisted of four different saccade paradigms, each paradigm being consecutively performed twice (with a different sequence of events), always presented in the same order. The rationale for this non-random order was to perform the easiest task first, then to introduce additional tasks of progressively increasing complexity. Each paradigm consisted of 18 rightward and 18 leftward saccades, presented in a semi-random order. Short breaks were taken after each pair of paradigms, during which the instructions for the next paradigm were given. A calibration procedure was systematically performed 
before starting each new pair of paradigms. The recording session in its entirety did not exceed 20 minutes.

- Oculomotor tasks (Fig. 1)

Visually guided saccade task (VGST): A white central fixation point (FP) was initially presented at the center of the screen for $1500-2800 \mathrm{~ms}$. As it disappeared, a green target was simultaneously presented at a $10^{\circ}, 17^{\circ}$ or $24^{\circ}$ lateral position, either right or left, for $1000 \mathrm{~ms}$. The central FP was then turned on again, and a new trial began. Children were instructed to trigger saccades as accurately and as fast as possible from the central FP to the lateral target, then back to the central FP. This paradigm allowed for the triggering of reflexive visually guided saccades.

Delayed saccade task (DST): The same initial conditions were used as in the VGST, except that the central FP did not disappear at lateral target onset. Both the FP and target remained on for a 5000-7000 ms, then only the FP was turned off. Participants were instructed to trigger a targeting saccade at FP offset. This delayed task allowed for the triggering of more volitional visually guided saccades.

Memory-guided saccade task (MGST): The same conditions were used as in the DST, except that the peripheral target was flashed during $100 \mathrm{~ms}$. Participants were instructed to wait until FP offset (i.e. during the $5000-7000$ ms delay) before triggering a saccade to the remembered position of the flash. This task allowed for the triggering of volitional (i.e. memory-guided) non-visually guided saccades.

Antisaccade task (AST): The same conditions as in the VGST were used, but participants were instructed to trigger, as fast as possible, a saccade in the opposite direction to the lateral target. This task allowed for the triggering of volitional non-visually guided saccades and evaluation of the ability to inhibit reflexive saccades.

\section{Parameters and Statistical analysis}

Mean saccade latency (time from target onset to saccade triggering, defined by a $40^{\circ} / \mathrm{s}$ velocity threshold) was measured for all centrifugal saccades. Mean saccade gain (ratio of first saccade amplitude on target position) and the variability of saccade gain were measured in both VGST and DST for centrifugal saccades, and in the VGST for centripetal saccades. Mean and maximum saccade velocities were measured for all centrifugal saccades in the 
VGS, MGST and AST. Since saccade velocity varies with saccade amplitude, saccades were sorted according to their amplitude and classified into three groups: large saccades (20 to $\left.24^{\circ}\right)$, medium saccades (15 to $\left.19^{\circ}\right)$, and small saccades $\left(10\right.$ to $\left.14^{\circ}\right)$.

Saccade skewness (ratio of the duration of the acceleration period on total saccade duration) was measured for all centrifugal saccades, in all tasks. Since saccade skewness varies with saccade amplitude [19], saccade skewness was evaluated in different categories of saccade amplitudes, similar to the categories used for the evaluation of saccade velocities. A skewness index was defined as the antisaccade skewness expressed as a percentage of visually guided saccade skewness.

Saccade error rate (percentage of misdirected saccades, i.e. saccades directed towards the target) was measured in the AST.

Statistical analysis

A Mann-Whitney test (U) was used in order to compare latencies, skewness, error rates, mean gains and gain variability between the 2 groups, and a Wilcoxon test was used for intra-group comparisons. A Kruskal Wallis test $(\mathrm{H})$ was used for the comparisons of saccade velocity and skewness for each of the 3 different amplitudes, and for each saccade parameter between the different tasks. This test was also used for comparisons of saccade skewness between dyspraxic and control groups and in each task. When significant differences were found, post hoc tests with Holm Bonferroni correction for multiple comparisons followed by Mann-Whitney $U$ tests were performed. Furthermore, we compared the skewness of large antisaccades measured in each individual subjects with DD to the mean value of the TD group using the one way t-test for single case $[20,21]$. The significance level for all inferential statistics was set at $5 \%$.

\section{Results}

Oculomotor results (Table 2)

\section{Saccade latencies}

Mean saccade latencies did not significantly differ between the two groups in the VGST, the DST and the AST, but were significantly increased in dyspraxic participants in the MGST $(U=4$; $p=0.00074)$.

Saccade gain 
Comparison between TD and DD groups showed that the mean gain of centrifugal saccades did not significantly differ in both VGST and DST, but that the variability of centrifugal saccade gain was significantly increased in dyspraxic participants in the VGST $(U=10$; $p=0.00091$ ) but not in the DST.

The main centripetal saccade gain, measured in the VGST, was not significantly different between the two groups, but the variability of centripetal saccade gain was significantly increased in the dyspraxic group $(U=17.5 ; p=0.0047)$.

Saccade velocities

Mean saccade velocity was not decreased in dyspraxic subjects in the VGST, for all saccade sizes. Conversely, in the MGST, mean velocity was significantly decreased $(H=35.03$; df 5; $p=0.00001)$ for large $(U=8 ; p=0.0029)$, medium $(U=4 ; p=0.0018)$ but not small saccades and in the AST $(H=33.3$ df $5 ; p=0.00001)$, for large $(U=7.5 ; p=0.0002)$, medium $(U=3.5 ; p=0.0014)$ but not small saccades.

Maximum saccade velocity was not decreased in dyspraxic subjects in the VGST for all saccade sizes. In the MGST, maximum saccade velocity was significantly decreased $(\mathrm{H}=$ 31.23; df 5; $p=0.00001)$ for medium ( $U=7 ; p=0.0025)$ but not for large and small saccades, and in the AST ( $H=26.41 \mathrm{df} 5 ; p=0.000074)$ only for large saccades $(U=12 ; p=0.0009)$.

\section{Saccade skewness}

Saccade skewness was first analyzed within each group (Fig. 2). In the TD group, saccade skewness showed a significantly inverse relationship with saccade amplitude, being smaller for larger saccades, this being observed in all saccade tasks: in the VGST $(H=22.53$; $d f$ 2; $p=0.000013)$, especially large vs small saccades $(U=0 ; p=0.00001)$, and large vs medium saccades $(U=11 ; p=0.011)$, in the DST $(H=18.57 \mathrm{df} 2 ; p=0.000093)$, especially large vs small saccades $(U=3.5 ; p=0.00002)$, medium vs small saccades $(U=14.5 ; p=0.014)$, in the MGST $(H=20.69$ df $2 ; p=0.000032)$, especially large vs small saccades $(U=1 ; p=0.00001)$, medium vs small saccades $(U=14 ; p=0.014)$ and in the AST $(H=9.35$ df $2 ; p=0.0093)$, especially large vs small saccades $(U=17.5 ; p=0.0023)$. Comparisons between saccade tasks $(H=83.45 ;$ df 11 ; $\mathrm{p}=0.00001$ ) did not reveal significant differences between VGST and DST, and between MGST and AST, but significant differences were observed between VGST and MGST (large saccades: $U=12 ; p=0.0014$; medium saccades: $U=13 ; p=0.0018$ ), between VGST and AST (medium saccades: $U=25 ; p=0.02$; small saccades: $U=12.5 ; p=0.0016$ ) and between DST and MGST (large saccades: $U=22 ; p=0.02$; medium saccades: $U=14 ; p=0.0023$ ) and between DST 
and AST (small saccades: $U=14 ; p=0.0023$ ). In summary, saccade skewness was influenced by saccade amplitude, being smaller for larger saccades, and by saccade task, being smaller for non-visually (MGST and AST) than for visually guided (VGST, DST) saccades (Fig.2).

In the DD group, a weaker relationship between saccade skewness and saccade amplitude was observed, being significant only in the DST $(H=10.61$, df 2, $p=0.005)$, for large vs small saccades $(U=0 ; p=0.0014)$ and in the AST $(H=11.21$; df $2 ; p=0.0037)$, for large vs small saccades $(U=3 ; p=0.001)$. Comparison between saccade tasks $(H=77.07 ;$ df $11 ; p=0.00001)$ found no difference between VGST and DST, and between MGST and AST (Fig. 2). However, saccade skewness was significantly different between VGST and MGST for all saccade sizes (large saccades: $U=0 ; p=0.0045$; medium saccades: $U=2 ; p=0.0018$; small saccades $U=2$; $p=0.002$ ), between VGST and AST (large saccades: $U=0 ; p=0.00007$; medium saccades: $U=0$; $p=0.0001$; small saccades: $U=0 ; p=0.0002$ ), and between DST and MGST (medium saccades: $\mathrm{U}=0 ; p=0.0076$; small saccades: $\mathrm{U}=1 \mathrm{p}=0.0078$ ) and between DST and AST (medium saccades: $U=0 ; p=0.00089$; small saccades: $U=0 ; p=0.0025$ ).

Comparison of saccade skewness between control and dyspraxic subjects revealed significant differences in the VGST for small saccades only $(H=35.95$, df $5 ; p=0.00001$, small saccades: $U=13 ; p=0.0025)$, no significant differences in the $D S T$, significant differences in the MGST for medium and small saccades $(H=42.04$, df 5; $p=0.00001$, medium saccades: $U=0 ; p=0.0001$; small saccades: $U=0 ; p=0.0001$ ), and significant differences in the AST for large, medium and small saccades $(H=46.35$ df 5; $p=0.00001$; large saccades: $U=0 ; p=0.0002$; medium saccades: $U=0 ; p=0.00036$; small saccades: $U=0 ; p=0.002$ ). In addition, we checked that AS skewness was decreased compared to VGS skewness in each DD (Fig 3), and that large antisaccade skewness of each DD subject was significantly larger than the mean value of the TD (Table 3).

The comparison of the skewness index (antisaccade skewness expressed as a percentage of visually guided saccade skewness) between both groups of participants ( $\mathrm{H}=41.07 \mathrm{df}$ $5 ; p=0.00001)$ confirmed that saccade skewness had a lower value in the AST than in the VGST and revealed a significantly greater skewness index in dyspraxic subjects than in controls (large saccades: $U=0 ; p=0.000015$; medium saccades: $U=0 ; p=0.00035$ and small saccades $U=2 p=0.0083$ )(Fig 4$)$. Since the antisaccade task was systematically performed at the end of the recording session, a decreased level of alertness in dyspraxic subjects might 
have resulted in decreased saccade velocities and therefore altered antisaccade skewness. We therefore measured saccade skewness of each centripetal saccades performed immediately after correct antisaccades, in both groups (Fig 5). The results showed that, in dyspraxic subjects, these centripetal saccades were significantly less skewed than the immediately preceding antisaccade $(p<0.01)$, and were not significantly different from similar centripetal saccades performed by control subjects $(p>0.05)$. These results thus enable to rule out a decreased arousal in dyspraxic patients.

\section{Antisaccade error rate}

Antisaccade error rates (ER) were significantly increased in the dyspraxic group $(p=0.0058)$. The analysis of individual data revealed an increased ER (i.e. out of $\mathrm{Cl}$ [6.54-12.65]) in 9 out of 11 dyspraxic subjects.

\section{Discussion}

This study is the first to analyze in detail saccadic eye movements in subjects with DD. The most striking and unexpected finding was the observation of markedly abnormal saccade dynamics in a group of dyspraxic adolescents. Other impairments consisted of increased memory-guided saccade latencies, increased error rates in the AST and increased variability of visually guided saccade amplitudes.

In order to interpret these results, a brief overview of the neurophysiology of saccades is warranted [10]. Saccades are controlled by a neural network that involves frontal and parietal cortical areas, subcortical structures (basal ganglia and superior colliculus), the cerebellum, and the saccade generator in the brainstem [10, 22]. Cortical oculomotor areas are mainly engaged in target localization and selection, and in decision-making processes. The parietal eye field (PEF) is more concerned with the control of reflexive-like saccades, whereas the frontal eye field (FEF) mainly controls the triggering of more purposive saccades [23]. A prefrontal area (Brodmann's area 46) is especially important when a reflexive saccade needs to be canceled $[24,25]$. The basal ganglia, located on frontal efferent pathways, are able to modulate purposive saccadic signals with respect to cognitive factors, such as target selection, target prediction, short-term memory, or motivation [26]. The superior colliculus, located between cortical areas and brainstem centers, has a potential influence on all saccade parameters (saccade latency, velocity, and amplitude) [11, 27]. Cerebellar structures involved in saccade control are principally the posterior vermis and the underlying fastigial 
oculomotor region (FOR). The main role of these structures is to maintain a high level of saccade accuracy on a trial-to-trial basis and in the long term [6]. Finally, the saccade generator contains burst neurons that encode saccade velocity [10].

Our dyspraxic group showed strong evidence for a frontal lobe dysfunction. Increased memory-guided saccade latencies but normal visually guided saccade latencies is typically observed after FEF inactivation [28, 29]. A decreased ability to cancel reflexive saccades (i.e. increased antisaccade error rate) is another frontal lobe symptom ascribed to an affected dorsolateral prefrontal cortex [24, 30, 31].

Besides cortical symptoms, we observed in most dyspraxic subjects unambiguous signs of cerebellar dysfunction. Cerebellar dysfunction typically results in the occurrence of intermingled hypometric and hypermetric saccades, expressed by an increased variability of saccade gain [11], as demonstrated in our dyspraxic group in the VGST and the DST. Hence, these results are in agreement with our initial hypothesis of both frontal lobe and cerebellar symptoms in dyspraxic children.

The analysis of saccade velocity did not initially appear as a relevant parameter. However, the observation of slowed saccades in the DD group, especially in the MGST and the AST, led us to introduce the analysis of saccade dynamics. It appeared that saccade slowing was related to prolonged deceleration phases, but relatively unaffected initial velocities, resulting in more skewed velocity profiles.

During a normal saccade, a powerful acceleration leads to peak eye velocity, immediately followed by a deceleration phase. For visually guided saccades of average amplitudes (e.g. $\left.10-12^{\circ}\right)$, both phases are of equal length [19]. If saccade velocity is plotted against time, an approximately symmetric (non-skewed) curve, centered on peak eye velocity, is obtained. Saccade skewness, defined as the duration of the acceleration phase divided by total saccade duration, refers to the more or less symmetrical aspect of this curve. It is mainly influenced by saccade amplitude and presence or absence of a visual cue at the saccadic goal [19]. For visually guided saccades of average amplitudes, its value is close to 0.50 [19]. The duration of the deceleration phase increases as saccade amplitude increases, resulting in asymmetrical velocity profile and saccade skewness below 0.50 . For saccades of similar amplitudes, the duration of the deceleration is longer in non-visually guided saccades, such as antisaccades and memory-guided saccades. Theoretically, saccades of increased skewness refer to saccades in which both phases are of unequal duration, but a large majority of the 
abnormal skewnesses corresponds to increased duration of the deceleration phase. Therefore, increased skewness, as used henceforth, most often refers to a value below 0.50 . The analysis of saccade skewness in our control group is in good accordance with previous data: saccade skewness increased with saccade amplitudes (Fig. 2) and was larger in the non-visually guided (MGST and AST), than in the visually guided (VGST and DST) tasks. A similar trend was observed in the dyspraxic group: saccades of larger amplitude tended to be more skewed, and the most skewed saccades were observed in the MGST and the AST. However, whereas the modulation with saccade amplitude tended to be slightly less than in control subjects, the influence of saccadic type was markedly more pronounced: saccades were strikingly more skewed in the AST than in the VGST, with a marked increased duration of the deceleration phase.

A decreased state of alertness may lead to saccades of decreased velocities with an altered velocity profile [19]. We however ruled out this hypothesis by showing that, in the antisaccade task, centripetal saccades performed immediately after correct antisaccades were not only less skewed than the immediately preceding antisaccade but also not different from those performed by control subjects.

We then hypothesized that this observation could be ascribed to an impaired cerebellar control, since the increased saccade duration was mainly due to an increased deceleration phase. However, the oculomotor vermis and the underlying FOR seem to exert control over all types of saccades, without specificity for visually or non visually guided saccades [32, 33]. The origin of this pattern could therefore arise from another cerebellar structure in which such selectivity occurs, or from an upstream structure. Few studies have shown that the cerebellar control over saccades may also include paravermal areas that receive frontal efferent signals via the pontine nuclei and the nucleus reticularis tegmenti pontis (NRTP) [34, 35]. An experimental dysfunction of these areas induces an increased variability of saccade accuracy without alteration of the mean saccadic gain [35], which is what we have observed in our patients with DD. However, since the effect on saccade skewness has not been analyzed, this hypothesis remains speculative.

As stated above, a larger impairment of non-visually saccades compared to visually guided saccades suggests an FEF dysfunction [36, 37]. However, cortical areas exert little, if any, influence on saccade velocity profiles. Saccade velocity profiles were analyzed after either 
micro [28] or large [38] FEF inactivations and found decreased saccade velocities without significant alterations of saccade skewness [ 28 and personal communication].

The SC and the NRTP are interposed between oculomotor cortical areas and the cerebellum [39]. An SC dysfunction is an unlikely hypothesis, since its inactivation results in a homogenous decrease of saccade velocity, affecting equally initial and later phases of saccades [11]. Conversely, NRTP inactivation results in markedly abnormal saccade velocity profiles with increased deceleration phases [40]. Although more studies are needed to confirm our hypothesis, we propose that the particular velocity profile observed in the children with DD could result from a flawed communication between the frontal cortices and the cerebellum at the pontine level.

We cannot, however, conclude with certainty that this anomaly is unique to DD and independent of the psychiatric and neurodevelopmental comorbidities that were idiosyncratic to the sample (Table 1). The cerebellum is characterized by an extensive connectivity network, which comprises the basal ganglia and distributed regions of the cerebral cortex. Accumulating evidence from anatomical, structural and functional imaging and lesion studies provides a substrate by which cerebellar abnormalities are involved in autism spectrum disorder [41, 42], schizophrenia [43], depressive disorders [44], ADHD [45], specific language impairment [46] and specific learning disorders [47]. These abnormalities affect grey matter, Purkinje cells, and cerebellar volume (for a review see 47). Furthermore, commonalities can be found in the putative networks involved, which is not surprising given the comorbidity between the disorders, but no overlap in the affected regions were found $[44,47]$. Moreover, given the clinical heterogeneity of the aforementioned disorders (following the example of autism spectrum disorders) which match the majority of those in our sample (Table 1), the direct link between cerebellar structure and function and the symptoms exhibited in each disorder has yet to be established. Until now, the analyses of saccade velocities in patients with autism spectrum disorders [48] or schizophrenia $[49,50]$ have not shown similar patterns of saccade abnormalities. Beyond the diversity in diagnoses concerning our sample (Cf. table 1), patients present diagnostic homogeneity of DD associated with what we consider an unusual saccade velocity pattern.

From a neuro-psychiatric point of view, our aim is to determine the behavioral correlates of these impaired saccade dynamics through the investigation of a large number of children 
with DD. A correlation between the present oculomotor finding and a given profile could address the complexity of this neurodevelopmental disorder. 


\section{References}

1. Vaivre-Douret L. Developmental coordination disorders: state of art. Neurophysiol Clin. 2014;44:13-23.

2. Vaivre-Drouet L, Lalanne C, Cabrol D, Ingster-Moati I, Falissard B, Golse B. Identification of diagnostic criteria of developmental coordination disorder (DCD) or developmental dyspraxia subtypes. Neurospychiatrie de l'Enfance et de l'Adolescence. 2011a;59:443-53.

3. Vaivre-Drouet L, Lalanne C, Ingster-Moati I, Boddaert N, Cabrol D, Dufier JL, et al. Subtypes of developmental dyspraxia and developmental coordination disorder (DCD): research on their nature and etiology. Developmental Neuropsychology. 2011b;36:1-29.

4. Xavier J, Tilmont E, Bonnot O. Children's synchrony and rhythmicity in imitation of peers: toward a developmental model of empathy. J Physiol Paris. 2013;107:291-7.

5. Bo J, Lee CM. Motor skill learning in children with Developmental Coordination Disorder. Res Dev Disabil. 2013;34:2047-55.

6. Kheradmand A, Zee DS. Cerebellum and ocular motor control. Front Neurol. 2011;2:1-15.

7. Langaas T, Mon-Williams M, Wann JP, Pascal E, Thompson C. Eye movements, prematurity and developmental co-ordination disorder. Vision Res. 1998;38:1817-26.

8. Lahuis BE, Van Engeland H, Cahn W, Caspers E, Van der Geest JN, Van der Gaag RJ, Kemner C. Smooth pursuit eye movement (SPEM) in patients with multiple complex developmental disorder (MCDD), a subtype of the pervasive developmental disorder. World J Biol Psychiatry. 2009;10:905-12.

9. Robert MP, Ingster-Moati I, Albuisson E, Cabrol D, Golse B, Vaivre-Douret L. Vertical and horizontal smooth pursuit eye movements in children with developmental coordination disorder. Dev Med Child Neurol. 2014;56:595-600. 
10. Gaymard B. Cortical and sub-cortical control of saccades and clinical application. Rev Neurol (Paris). 2012;168:734-40.

11. Quaia C, Lefèvre P, Optican LM. Model of the control of saccades by superior colliculus and cerebellum. J Neurophysiol. 1999;82:999-1018.

12. American Psychiatric Association. Diagnostic and Statistical Manual of mental disorders. $5^{\text {th }}$ edn. Arlington, VA: American Psychiatric Publishing, 2013.

13. Henderson SE, Sugden DA. Movement assessment battery for children. London: Psychological Corporation. 1992.

14. Brannigan GG, Decker SL. The Bender-Gestalt II. Am J Orthopsychiatry. 2006;76:10-2.

15. Maslow P, Frostig M, Lefever DW, Whittlesey JR. The Marianne Frostig developmental test of visual perception, 1963 Standardization. Percept Mot Skills. 1964;19:463-99.

16. Blote AW, Hamstra-Bletz L. A longitudinal study on the structure of handwriting. Percept Motor Skills. 1991;72:983-94.

17. Wechsler D. Wechsler intelligence scale for children-Fourth Edition (WISC-IV). San Antonio, TX: The Psychological Corporation. 2003.

18. Wechsler D. Wechsler adult intelligence scale-Fourth Edition (WAIS-IV). San Antonio, TX: NCS Pearson. 2008

19. Smit AC, Van Gisbergen JA, Cools AR. A parametric analysis of human saccades in different experimental paradigms. Vision Res. 1987;27:1745-62.

20. Crawford JR, Garthwaite PH. Investigation of the single case in neuropsychology: Confidence limits on the abnormality of test scores and test score differences. Neuropsychologia. 2002;40,1196-208. 
21. Crawford JR, Howell DC. Comparing an individual's test score against norms derived from small samples. The Clinical Neuropsychologist. 1998;12,482-86.

22. Leigh RJ, Kennard C. Using saccades as a research tool in the clinical neurosciences. Brain. 2004;127:460-77.

23. Gaymard B, Pierrot-Deseilligny C. Neurology of saccades and smooth pursuit. Curr Opin Neurol. 1999;12:13-9.

24. Ploner CJ, Gaymard BM, Rivaud-Péchoux S, Pierrot-Deseilligny C.The prefrontal substrate of reflexive saccade inhibition in humans. Biol Psychiatry. 2005;57:1159-65.

25. Pierrot-Deseilligny C, Müri RM, Ploner CJ, Gaymard B, Demeret S, Rivaud-Pechoux S. Decisional role of the dorsolateral prefrontal cortex in ocular motor behaviour. Brain. 2003;126:1460-73.

26. Hikosaka O, Takikawa Y, Kawagoe R. Role of the basal ganglia in the control of purposive saccadic eye movements. Physiol Rev. 2000;80:953-78.

27. Munoz DP, Dorris MC, Paré $M$, Everling S. On your mark, get set: brainstem circuitry underlying saccadic initiation. Can J Physiol Pharmacol. 2000;78:934-44.

28. Dias EC, Segraves MA. Muscimol-induced inactivation of monkey frontal eye field: effects on visually and memory-guided saccades. J Neurophysiol. 1999;81:2191-214.

29. Sommer MA, Tehovnik EJ. Reversible inactivation of macaque dorsomedial frontal cortex: effects on saccades and fixations. Exp Brain Res. 1999;124:429-46.

30. Pierrot-Deseilligny C, Rivaud S, Gaymard B, Agid Y. Cortical control of reflexive visuallyguided saccades. Brain. 1991;114:1473-85. 
31. Condy C, Rivaud-Péchoux S, Ostendorf F, Ploner CJ, Gaymard B. Neural substrate of antisaccades: role of subcortical structures. Neurology. 2004;63:1571-8.

32. Kojima Y, Soetedjo R, Fuchs AF. Behavior of the oculomotor vermis for five different types of saccade. J Neurophysiol. 2010;104:3667-76.

33. Ohtsuka K, Noda H. Burst discharges of fastigial neurons in macaque monkeys are driven by vision- and memory-guided saccades but not by spontaneous saccades. Neurosci Res. $1992 ; 15: 224-8$.

34. Ron S, Robinson DA. Eye movements evoked by cerebellar stimulation in the alert monkey. J Neurophysiol. 1973;36:1004-22.

35.Ohki M, Kitazawa H, Hiramatsu T, Kaga K, Kitamura T, Yamada J, Nagao S. Role of primate cerebellar hemisphere in voluntary eye movement control revealed by lesion effects. J Neurophysiol. 2009;101:934-47.

36. Gaymard B, Ploner CJ, Rivaud-Péchoux S, Pierrot-Deseilligny C. The frontal eye field is involved in spatial short-term memory but not in reflexive saccade inhibition. Exp Brain Res. 1999;129:288-301.

37. Rivaud S, Müri RM, Gaymard B, Vermersch Al, Pierrot-Deseilligny C. Eye movement disorders after frontal eye field lesions in humans. Exp Brain Res. 1994;102:110-20.

38. Peel TR, Johnston K, Lomber SG, Corneil BD. Bilateral saccadic deficits following large and reversible inactivation of unilateral frontal eye field. J Neurophysiol. 2014;111:415-33.

39. Thier $P$, Möck $M$. The oculomotor role of the pontine nuclei and the nucleus reticularis tegmenti pontis. Prog Brain Res. 2006;151:293-320. 
40. Kaneko CR, Fuchs AF. Effect of pharmacological inactivation of nucleus reticularis tegmenti pontis on saccadic eye movements in the monkey. J Neurophysiol. 2006;95:3698711.

41. Becker EB, Stoodley CJ. Autism spectrum disorder and the cerebellum. Int Rev Neurobiol. 2013;1;113:1-34.

42. Courchesne E, Mouton PR, Calhoun ME, Semendeferi K, Ahrens-Barbeau C, Hallet MJ, et al. Neuron number and size in prefrontal cortex of children with autism. JAMA. 2011;306, 2001-2010.

43. Bernard JA, Mittal VA. Cerebellar-motor dysfunction in schizophrenia and psychosis-risk: the importance of regional cerebellar analysis approaches. Front Psychiatry. 2014;5(160.10):3389.

44. Phillips JR, Hewedi DH, Eissa AM, Moustafa AA. The cerebellum and psychiatric disorders. Frontiers in public health. 2015;34. Doi:10.1016/B978-0-12-418700-9.000011-0

45. Stoodley CJ. Distinct regions of the cerebellum show gray matter decreases in autism, ADHD, and developmental dyslexia. Distributed Networks-New Outlooks on Cerebellar Function. 2015 Jul 3:89.

46. Hodge SM, Makris N, Kennedy DN, Caviness Jr VS, Howard J, McGrath L, Steele S, Frazier JA, Tager-Flusberg H, Harris GJ. Cerebellum, language, and cognition in autism and specific language impairment. Journal of Autism and Developmental Disorders. 2010;1;40:300-16.

47. Manto MU, Jissendi P. Cerebellum: links between development, developmental disorders and motor learning. Frontiers in Neuroanatomy. 2012 6(January), 1. http://doi.org/10.3389/fnana.2012.0000 
48. Schmitt LM, Cook EH, Sweeney JA, Mosconi MW. Saccadic eye movement abnormalities in autism spectrum disorder indicate dysfunctions in cerebellum and brainstem. Mol Autism. 2014;5:47.

49. Cui H, Liu XH, Wang KY, Zhu CY, Wang C, Xie XH. Association of saccade duration and saccade acceleration/deceleration asymmetry during visually guided saccade in schizophrenia patients. PLoS One. 2014;9(5):e97308.

50. Gurvich CT, Fitzgerald PB, Georgiou-Karistianis N, White OB. Saccadic impairment in schizophrenia with prominent negative symptoms. Neuroreport. 2008;19:1435-9. 


\section{Captions of figures}

Figure 1: Saccade paradigms. A: Visually guided saccade task. B: Delayed saccade task. C: Memory-guided saccade task. D: Antisaccade task.

Figure 2: Saccade skewness according to saccade task and saccade amplitude in Control and Dyspraxic subjects.VGS: Visually guided saccades; DS: Delayed saccades; MGS: Memoryguided saccades; AS: Antisaccades.

Figure 3: Individual values of saccade skewness in TD and DD groups, in large visually guided saccades and large antisaccades.AS: Antisaccades; VGS: Visually guided saccades; DD: Subjects with developmental dyspraxia; TD: Typically developing subjects.

Figure 4: skewness index in Control and Dyspraxic subjects.

Skewness index is defined as antisaccade skewness expressed as a percentage of visually guided saccade skewness.

Figure 5: skewness of large visually-guided, large antisaccades and centripetal saccades performed after the large antisaccades, in Control and Dyspraxic subjects. VGS: Visually guided saccades; AS: Antisaccades; CP PS: centripetal prosaccades. 

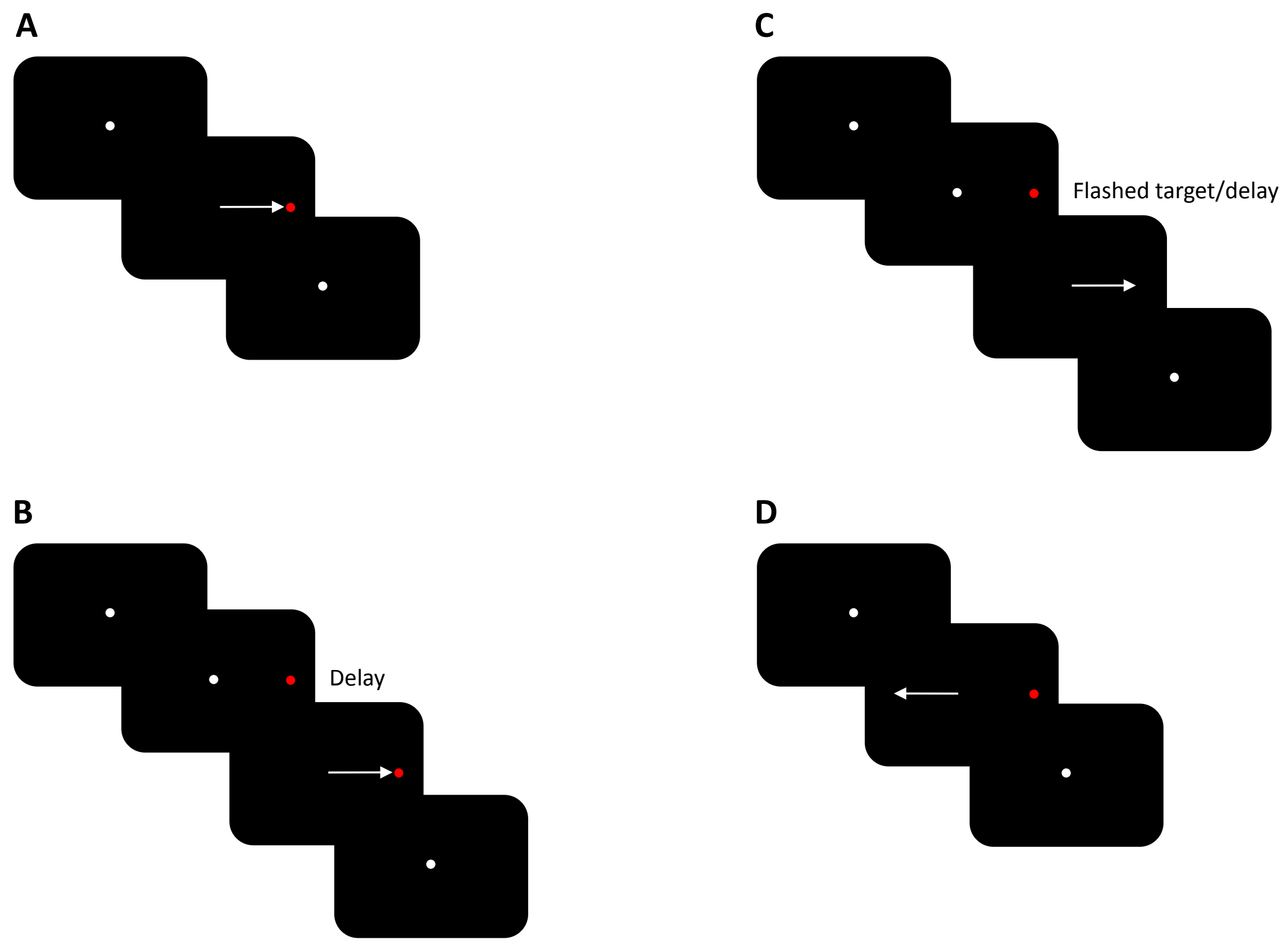

Figure 1 
skewness

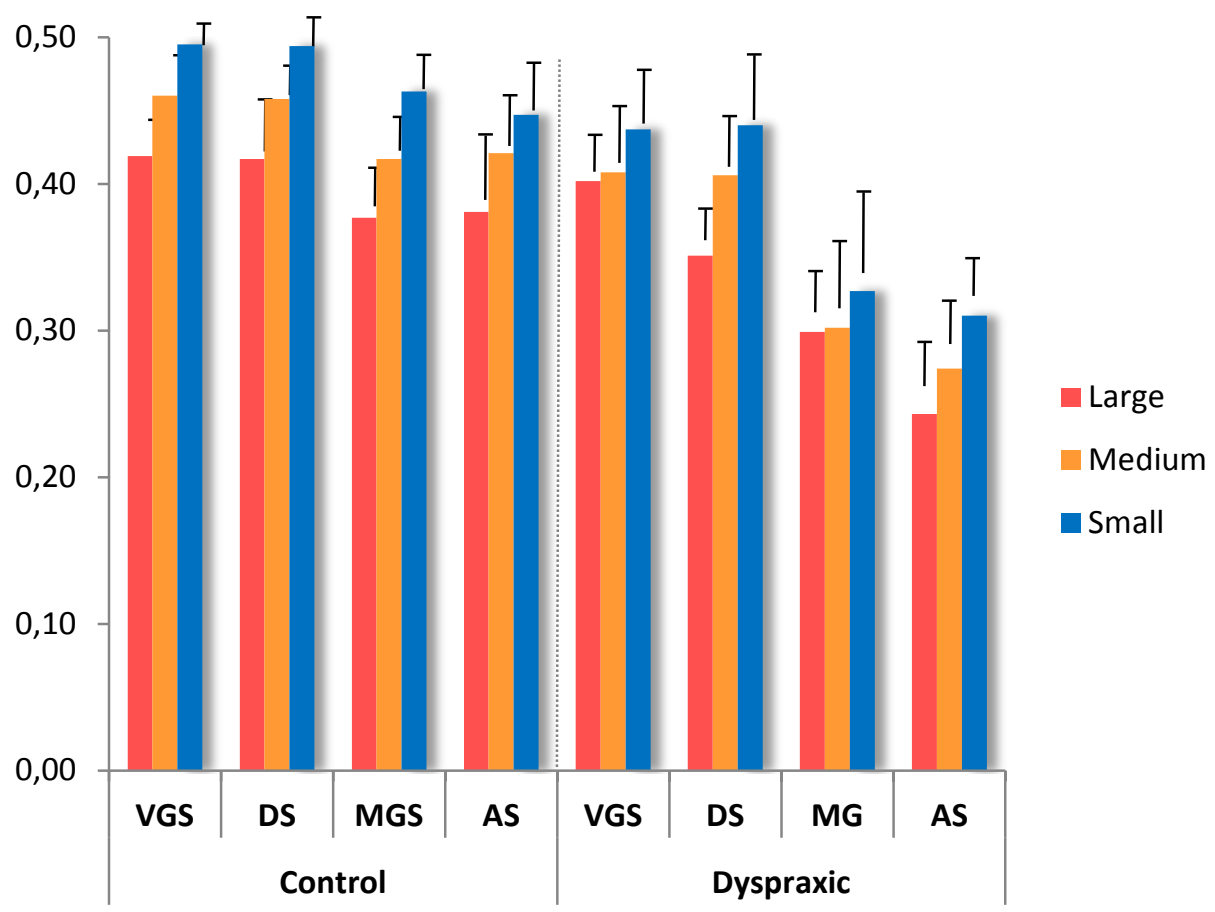

Figure 2 


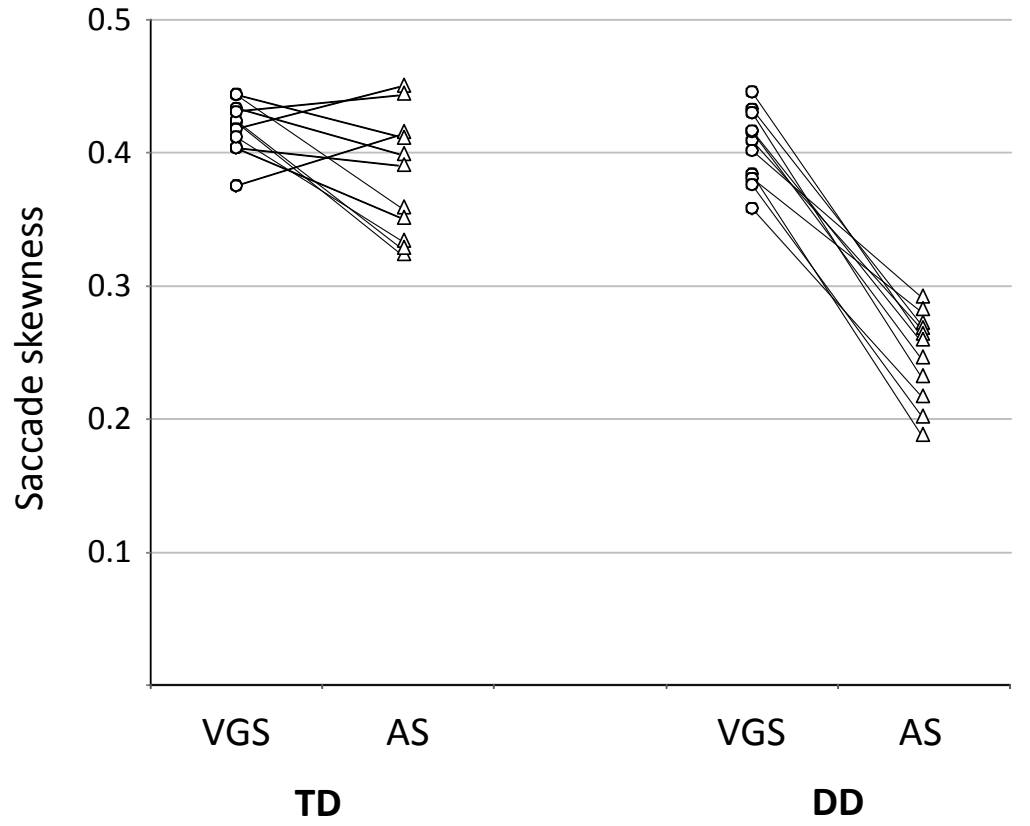

Figure 3 


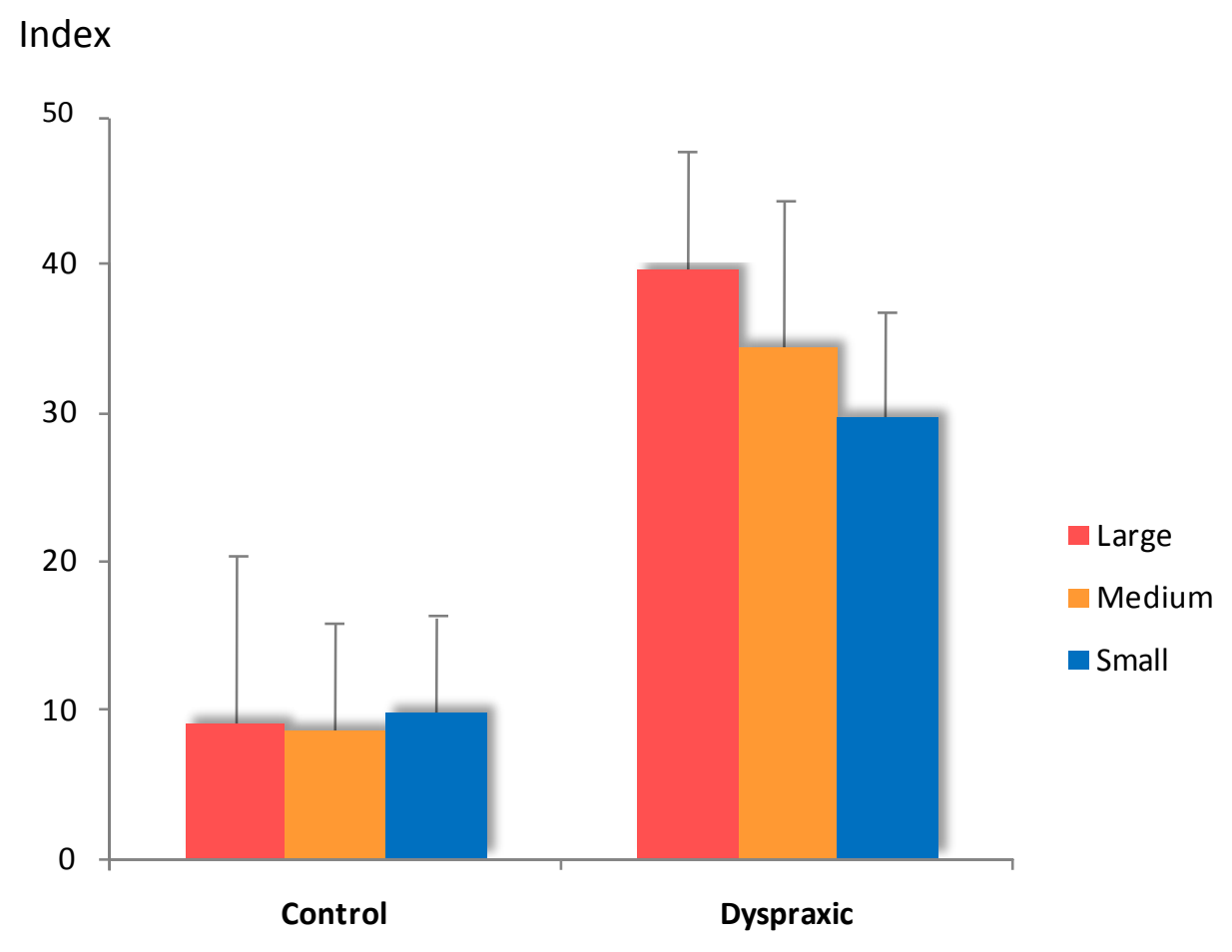

Figure 4 
Skewness

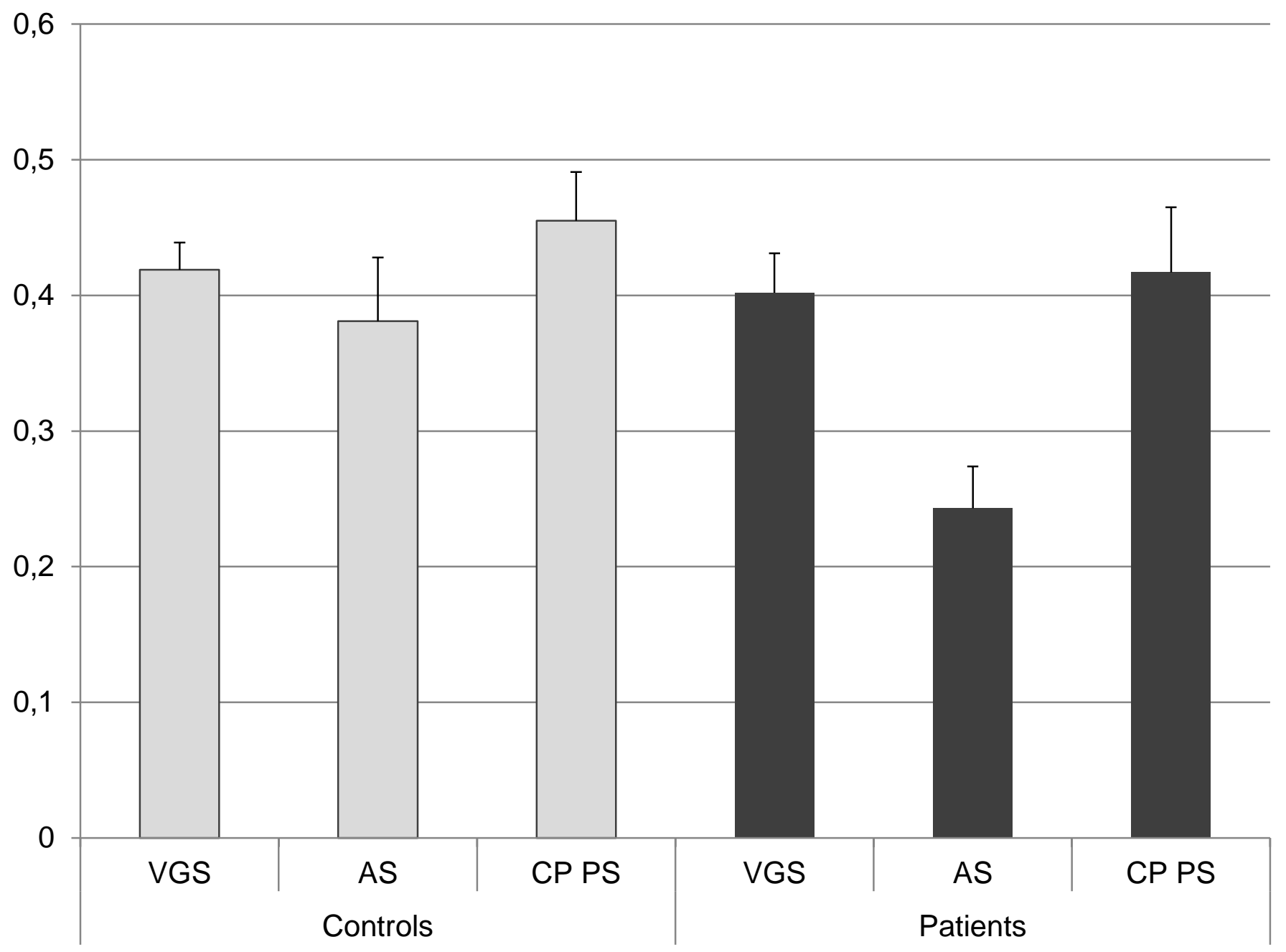

Figure 5 


\begin{tabular}{|c|c|c|c|c|c|c|c|}
\hline \multirow[b]{2}{*}{$\mathbf{N}$} & \multirow[b]{2}{*}{ Age } & \multirow[b]{2}{*}{ Sex } & \multicolumn{3}{|c|}{ DSM IV-TR Diagnostic criteria } & \multirow[b]{2}{*}{$\begin{array}{l}\text { Psychiatric } \\
\text { comorbidity }\end{array}$} & \multirow[b]{2}{*}{ Comments on specific assessments } \\
\hline & & & $\begin{array}{l}\text { Motor } \\
\text { signs } \\
\text { (A) }\end{array}$ & $\begin{array}{l}\text { Daily } \\
\text { impact } \\
\text { (B) }\end{array}$ & $\begin{array}{l}\text { Age of } \\
\text { onset } \\
\text { (C) }\end{array}$ & & \\
\hline 1 & 16 & $\mathrm{~F}$ & ++ & +++ & $<3$ years & $\begin{array}{l}\text { Autism Spectrum } \\
\text { Disorder }\end{array}$ & $\begin{array}{l}\text { WISC-IV assessment showed important } \\
\text { discrepancy between subscales }(\mathrm{VCl}=101 ; \mathrm{PRI}=88 \text {; } \\
\text { WMI=91; } \mathrm{PSI}=71) \text {. Poor performances at } \mathrm{M}-\mathrm{ABC} \\
\text { and Bender-Gestalt test }\end{array}$ \\
\hline 2 & 17 & $\mathrm{M}$ & +++ & +++ & $<5$ years & $\begin{array}{l}\text { Reading disorder } \\
\text { Disorder of written } \\
\text { expression }\end{array}$ & $\begin{array}{l}\text { WISC-III assessment showed important } \\
\text { discrepancy between VIQ }=95 \text { and } P I Q=67\end{array}$ \\
\hline 3 & 17 & $\mathrm{M}$ & ++ & ++ & $<5$ years & $\begin{array}{l}\text { ADHD } \\
\text { Schizophrenia } \\
\text { Disorder of written } \\
\text { expression }\end{array}$ & $\begin{array}{c}\text { WISC-III assessment showed important } \\
\text { discrepancy between } \text { VIQ }=101 \text { and } P I Q=83 . \\
\text { BHK test and M-ABC showed graphomotor } \\
\text { difficulties. }\end{array}$ \\
\hline 4 & 13 & $\mathrm{M}$ & ++ & +++ & $<5$ years & Reading disorder & $\begin{array}{c}\text { WISC-IV assessment showed important } \\
\text { discrepancy between subscales (VCl=78; PRI=84; } \\
\text { WMI=58; PSI=69). } \\
\text { Psychomotor assessment showed poor } \\
\text { performances on motor and perceptive tasks. }\end{array}$ \\
\hline 5 & 14 & $\mathrm{~F}$ & ++ & +++ & $<5$ years & $\begin{array}{l}\text { Reading disorder } \\
\text { Major depression } \\
\text { with psychotic } \\
\text { features }\end{array}$ & $\begin{array}{c}\text { WISC-IV assessment showed important } \\
\text { discrepancy between subscales (VCI=96; PRI=77; } \\
\text { WMI=85; PSI=83). } \\
\text { Delayed perceptive skills at Frostig. }\end{array}$ \\
\hline 6 & 15 & $\mathrm{M}$ & +++ & ++ & $<3$ years & $\begin{array}{l}\text { SLI and reading } \\
\text { disorder. Disorder of } \\
\text { written expression }\end{array}$ & $\begin{array}{l}\text { Psychomotor assessment showed poor handing } \\
\text { coordination, resulting in clumsiness and slow } \\
\text { motor performances, and severe difficulties for } \\
\text { graphic tasks. }\end{array}$ \\
\hline 7 & 13 & $\mathrm{~F}$ & ++ & ++ & $<3$ years & $\begin{array}{l}\text { Mathematics } \\
\text { disorder }\end{array}$ & $\begin{array}{l}\text { Psychomotor assessment showed poor } \\
\text { performances on motor and perceptive tasks. }\end{array}$ \\
\hline 8 & 16 & $M$ & +++ & +++ & $<3$ years & $\begin{array}{l}\text { Adjustment disorder } \\
\text { with mixed } \\
\text { disturbance of } \\
\text { emotions and } \\
\text { conduct }\end{array}$ & $\begin{array}{l}\text { WISC-IV assessment showed important } \\
\text { discrepancy between subscales (VCI=122; } \\
\text { PRI=107; WMI=86; PSI=97). } \\
\text { Psychomotor assessment showed poor } \\
\text { performances on visual-motor tasks. M-ABC } \\
\text { showed poor visual-motor coordination skills. }\end{array}$ \\
\hline 9 & 22 & $\mathrm{~F}$ & ++ & ++ & $<5$ years & $\begin{array}{l}\text { SLI and reading } \\
\text { disorder }\end{array}$ & $\begin{array}{l}\text { WISC-III assessment showed normal (low range) } \\
\text { intelligence (VIQ=80 and } \mathrm{PIQ}=75) .\end{array}$ \\
\hline 10 & 16 & M & +++ & +++ & $<3$ years & $\begin{array}{l}\text { Reading disorder } \\
\text { Mathematics } \\
\text { disorder } \\
\text { Schizophrenia }\end{array}$ & $\begin{array}{l}\text { WISC-III assessment showed borderline } \\
\text { intelligence, with important discrepancy between } \\
\qquad \text { VIQ }=81 \text { and } P I Q=63 \text {. } \\
\text { Psychomotor assessment (Rey complex figure } \\
\text { test) showed poor visuo-spatial skills and severe } \\
\text { difficulties for visual-constructive abilities. }\end{array}$ \\
\hline 11 & 17 & $\mathrm{~F}$ & ++ & ++ & $<3$ years & Schizophrenia & $\begin{array}{l}\text { Psychomotor assessment (M-ABC; Frostig) } \\
\text { showed poor performances on motor and } \\
\text { perceptive tasks. } \\
\text { WISC-IV assessment showed borderline } \\
\text { intelligence (VCI=76; PRI=77; WMI=79; PSI=83) }\end{array}$ \\
\hline
\end{tabular}


Table 1. Clinical characteristics of the patients with dyspraxia.

F=Female; M=Male; SLI=Specific Language Impairment; WISC-IV=Wechsler Intelligence Scale for Children (IV edition); VIQ=Verbal IQ; PIQ=Performance IQ; VCI=Verbal Comprehension Index; PRI =Perceptual Reasoning Index; WMI=Working Memory Index; PSI=Processing Speed Index; ADHD=Attention Deficit Hyperactivity Disorder; M-ABC=Movement Assessment Battery for Children; BHK= Beknopte Beoordelingsmethode voor Kinderhandschriften. 


\begin{tabular}{|c|c|c|c|c|c|}
\hline & & & & Control Group & Dyspraxic group \\
\hline \multirow{11}{*}{ VGST } & Latency & & $\mathrm{m}$ & 189 & 209 \\
\hline & \multirow{4}{*}{ Gain } & \multirow{2}{*}{ CF } & $\mathrm{m}$ & 0.95 & 0.92 \\
\hline & & & $\mathrm{sd}$ & 0.06 & $0.09 * *$ \\
\hline & & \multirow{2}{*}{$\mathrm{CP}$} & $\mathrm{m}$ & 0.97 & 0.93 \\
\hline & & & $\mathrm{sd}$ & 0.06 & $0.09 * *$ \\
\hline & \multirow{6}{*}{ Velocity } & \multirow{3}{*}{ Max } & $\mathrm{L}$ & 530 & 461 \\
\hline & & & $M$ & 511 & $434^{*}$ \\
\hline & & & $\mathrm{S}$ & 410 & 378 \\
\hline & & \multirow{3}{*}{ Mean } & $\mathrm{L}$ & 305 & $264 *$ \\
\hline & & & $M$ & 288 & $242 *$ \\
\hline & & & $S$ & 226 & 210 \\
\hline \multirow{3}{*}{ DST } & Latency & & $\mathrm{m}$ & 315 & 372 \\
\hline & \multirow{2}{*}{ Gain } & \multirow{2}{*}{$\mathrm{CF}$} & $\mathrm{m}$ & 0.97 & 0.98 \\
\hline & & & $\mathrm{sd}$ & 0.07 & 0.09 \\
\hline \multirow{7}{*}{ MGST } & Latency & & $\mathrm{m}$ & 297 & $383 * *$ \\
\hline & \multirow{6}{*}{ Velocity } & \multirow{3}{*}{ Max } & $\mathrm{L}$ & 481 & $263^{*}$ \\
\hline & & & $\mathrm{M}$ & 450 & $244 * *$ \\
\hline & & & $\mathrm{S}$ & 391 & $211 * *$ \\
\hline & & \multirow{3}{*}{ Mean } & $\mathrm{L}$ & 263 & $209 *$ \\
\hline & & & $M$ & 244 & $195 * *$ \\
\hline & & & $\mathrm{S}$ & 211 & $179 * *$ \\
\hline \multirow{8}{*}{ AST } & Latency & & $\mathrm{m}$ & 319 & 361 \\
\hline & \multirow{6}{*}{ Velocity } & \multirow{3}{*}{ Max } & $\mathrm{L}$ & 494 & $377 * *$ \\
\hline & & & $\mathrm{M}$ & 458 & $360 * *$ \\
\hline & & & $\mathrm{S}$ & 422 & $324^{*}$ \\
\hline & & \multirow{3}{*}{ Mean } & $\mathrm{L}$ & 260 & $195 * * *$ \\
\hline & & & $M$ & 249 & $186 * * *$ \\
\hline & & & $S$ & 228 & $173 * * *$ \\
\hline & ER & & $\%$ & 9.6 & $29 *$ \\
\hline
\end{tabular}

Table 2. Oculomotor data.

VGST: visually guided saccade task; DST: delayed saccade task; MGST: memory-guided saccade task; AST: antisaccade task. CF: centrifugal; CP: centripetal; ER: error rate. m: mean; L: large; M: medium; S: small. *: $p<0.05 ;^{* *}: p<0.005 ;^{* * *}$ : $p<0.0005$. 


\begin{tabular}{|c|c|c|c|c|}
\hline Patients & Skewness & $\mathrm{t}$ & $\mathrm{df}$ & $\begin{array}{c}\mathrm{p} \text { one } \\
\text { tailed }\end{array}$ \\
\hline DD1 & 0,185 & 3,993 & 10 & 0,001 \\
DD2 & 0,279 & 2,078 & 10 & 0,032 \\
DD3 & 0,259 & 2,485 & 10 & 0,016 \\
DD4 & 0,269 & 2,282 & 10 & 0,023 \\
DD5 & 0,266 & 2,343 & 10 & 0,021 \\
DD6 & 0,200 & 3,687 & 10 & 0,002 \\
DD7 & 0,243 & 2,811 & 10 & 0,009 \\
DD8 & 0,215 & 3,382 & 10 & 0,003 \\
DD9 & 0,256 & 2,546 & 10 & 0,015 \\
DD10 & 0,229 & 3,096 & 10 & 0,006 \\
DD11 & 0,269 & 2,282 & 10 & 0,023 \\
\hline
\end{tabular}

Table 3. Comparison of large antisaccade skewness in each dyspraxic subject (DD1 to DD11) to the mean antisaccade skewness of the control group (mean skewness in the control group: 0.381 , sd $0.047)[20,21]$. 